\title{
Design of a soft gamma-ray focusing telescope for the study of nuclear lines
}

C. M. Hubert Chen, Finn Erland Christensen, Fiona A. Harrison, Peter H. Mao, David L. Windt

C. M. Hubert Chen, Finn Erland Christensen, Fiona A. Harrison, Peter H. Mao, David L. Windt, "Design of a soft gamma-ray focusing telescope for the study of nuclear lines," Proc. SPIE 4851, X-Ray and Gamma-Ray Telescopes and Instruments for Astronomy, (11 March 2003); doi: 10.1117/12.461419

Event: Astronomical Telescopes and Instrumentation, 2002, Waikoloa, Hawai'i, United States 


\title{
Design of a soft-gamma-ray focusing telescope for the study of nuclear lines
}

\author{
C. M. Hubert Chen ${ }^{a}$, Finn E. Christensen ${ }^{b}$, Fiona A. Harrison ${ }^{a}$, Peter H. Mao ${ }^{a}$ \\ and David L. Windt ${ }^{c}$ \\ ${ }^{a}$ California Institute of Technology, Mail Code 220-47, Pasadena, CA 91125, USA \\ ${ }^{b}$ Danish Space Research Institute, \\ Juliane Maries Vej 30, Copenhagen Ø, DK-2100, Denmark \\ ${ }^{c}$ Columbia Astrophysics Laboratory, 550 West 120th Street, New York, NY 10027, USA
}

\begin{abstract}
We have studied the design of astronomical multilayer telescopes optimized for performance from 5 to $200 \mathrm{keV}$. This region of the spectrum contains important nuclear lines that are observable in supernovae and their remnants. The study of these lines can help to differentiate currently competing theories of supernova explosion. Our telescope design will enable us to measure the spectral lines of isotopes such as ${ }^{56} \mathrm{Ni}$ in Type Ia supernovae and ${ }^{44} \mathrm{Ti}$ in core-collapse remnants, as well as to observe active galactic nuclei at gamma-ray energies.

We considered the performances of multilayers of various material pairs, including $\mathrm{W} / \mathrm{Si}, \mathrm{Pt} / \mathrm{C}$ and $\mathrm{Ni}_{93} \mathrm{~V}_{7} / \mathrm{Si}$, as employed in conical-approximation Wolter I optics. We experimented with dividing the energy band of interest into several sections, and optimizing different groups of mirror shells within a single telescope for each smaller energy band. Different material pairs are also used for different energy bands, in order to obtain a higher overall performance. We also consider the significance of the energy bandwidth on the effectiveness of Joensen's parametrization of the multilayer thickness profile, and on the mirror performance within the band.
\end{abstract}

Keywords: Gamma-ray astrophysics, multilayer optimization, Type Ia supernova

\section{INTRODUCTION}

Focusing optics employed on the current generation of space telescopes, such as Chandra and XMM, have enabled us to study the cosmos with much improved signal-to-noise ratio and angular resolution at energies up to $10 \mathrm{keV}$. Several balloon experiments under development, including $\mathrm{HEFT}^{1}$ and $\mathrm{InFOC} \mu \mathrm{S},{ }^{2}$ demonstrate that one can extend the capability of focusing optics up to $100 \mathrm{keV}$ with multilayer coatings on the X-ray mirrors. Although no experiment is currently underway to realize a focusing telescope targeting beyond $100 \mathrm{keV}$, we believe that with the current state of the art technologies in X-ray optics and detector developments, such an endeavour is now possible for energy ranges up to about $200 \mathrm{keV}$.

The availability of a telescope in this energy range will provide astronomers a very useful tool to tackle many problems that are yet unsolved. A prominent example problem is that of understanding Type Ia supernova explosions. In a Type Ia supernova, ${ }^{56} \mathrm{Ni}$ is the dominant nuclear product, which decays through the emission of a $158 \mathrm{keV}$ gamma ray. There are currently several competing theories as to how Type Ia supernovae evolve, and they differ in their prediction of the time evolution and abundance of the elements emitted. With a multilayer focusing telescope operating in the 100 to $200 \mathrm{keV}$ range, one can measure the intensity of the 158-keV line of ${ }^{56} \mathrm{Ni}$ as it evolves with time, and thus differentiate the competing theories. To do this, a collecting area of $300 \mathrm{~cm}^{2}$ within a $30 \mathrm{keV}$ wide band about $158 \mathrm{keV}$ is needed. ${ }^{3}$ (This bandwidth is required to contain both the redshift and blueshift of the $158 \mathrm{keV}$ line, as the gamma rays are emitted from fast-moving ejecta in all directions.) Another important problem is the mapping of the 68 - and 78 -keV lines of ${ }^{44} \mathrm{Ti}$ in core-collapse supernova remnants, where most heavy elements in the universe are created through the process

Further author information: (Send correspondence to C.M.H.C.) C.M.H.C: E-mail: hubert@caltech.edu, Telephone: 16263956630 
Table 1. Geometric parameters for several focal length and radius range combinations.

\begin{tabular}{|l||c|c|c|c|}
\hline Focal Length $(\mathrm{m})$ & 15 & 20 & 25 & 30 \\
Minimum mirror radius $(\mathrm{cm})$ & 4 & 4 & 4 & 4 \\
Maximum mirror radius $(\mathrm{cm})$ & 30 & 30 & 35 & 35 \\
Minimum on-axis grazing angle (milliradians) & $2 / 3$ & 0.5 & 0.4 & $1 / 3$ \\
Maximum on-axis grazing angle (milliradians) & 5.0 & 3.75 & 3.5 & 2.917 \\
Number of 0.3 mm mirror shells & 276 & 330 & 416 & 459 \\
Weight of mirror substrate (kg/module) & 80.3 & 97.5 & 140. & 156. \\
\hline
\end{tabular}

of nucleosynthesis; this mapping requires a collecting area of $500-1000 \mathrm{~cm}^{2}$ at 68 and $78 \mathrm{keV} .^{3}$ The readers are referred to Harrison $\mathrm{et}^{3} \mathrm{l}^{3}$ for more details on the scientific requirements on a mission to study Type Ia supernovae and core-collapse remnants.

While soft-gamma-ray multilayer telescope designs have been made previously, e.g., in Christensen et al, ${ }^{4}$ we emphasize that our telescope design is distinct from them. For instance, Christensen et al only considered covering a broad, continuous energy band, as opposed to multiple narrow bands; it was aimed at larger satellite missions on the scale of XEUS, and thus did not restrict itself to extendable lengths of $30 \mathrm{~m}$ or so. It did not address the issue of minimizing weight, nor consider, in details, the trade-off between using tungsten-based and nickel-based multilayer coatings. All these topics are in the content that follows.

In this paper, we detail several telescope designs that will enable us to observe the ${ }^{56} \mathrm{Ni}$ line at $158 \mathrm{keV}$ and the ${ }^{44} \mathrm{Ti}$ lines at 68 and $78 \mathrm{keV}$. We describe our designing approach, with an emphasis on the multilayer coatings, and then present the telescope parameters with projected performance estimates in terms of the collecting area.

\section{THE DESIGN OF THE TELESCOPE}

Similar to most other X-ray focusing telescopes, we use the conical approximation to the Wolter I telescope as the basic mirror geometry. Using the balloon-borne High Energy Focusing Telescope ${ }^{1}$ as a basic platform for our design, we use thermally slumped glass as the mirror substrate, and put multilayer coatings on it to increase its reflectivity at large grazing angles and high energies. Each mirror shell is $20 \mathrm{~cm}$ long along the axis of the telescope, and $0.3 \mathrm{~mm}$ thick. We mount these shells on the outside of a 4-cm radius mandrel out to a maximum radius of $35 \mathrm{~cm}$ (30 cm for focal lengths of $20 \mathrm{~m}$ or shorter). While longer focal lengths give smaller on-axis grazing angles, which are desirable for high X-ray reflectivity, the actual focal length realisable is constrained by the mechanical stability of a long structure in space. Budget is also an issue as longer structures are more costly. We base our telescope design on (extendable) focal lengths between $15 \mathrm{~m}$ to $30 \mathrm{~m}$, which are within the reach of current spacecraft technologies. Table 1 shows the geometric parameters resulting from several sets of focal length and radius range combinations. The on-axis grazing angles range will be from $\frac{1}{3}$ to 2.92 milliradians. On the focal plane, a pixel detector made of cadmium zinc telluride (CdZnTe) is used. With the relatively high $\mathrm{X}$-ray stopping efficiency of CdZnTe, compared to most other detector materials, a thickness of about $5 \mathrm{~mm}$ suffices to collect over $77 \%$ of the focused X-ray at $158 \mathrm{keV}$. Current CdZnTe crystal sizes are limited to about $2.5 \mathrm{~cm}$ in length, but one can easily cover the desired focus area with an array of such detectors. On a typical mid-size explorer scale satellite mission, one can place up to seven of these $35-\mathrm{cm}$ radius telescope modules on the spacecraft to increase the collecting area. Alternatively, five large, 35-cm radius modules plus five small, 17 -cm radius modules can also fit.

Having laid out the geometric parameters of the telescope, we are set to describe the design of the multilayer coatings on the mirrors, which plays a major role in determining the reflectivity of the mirrors and the collecting area of the telescope. We have previously documented this design method in Mao et $\mathrm{al}^{5}{ }^{5}$ which the readers are referred for more details than the description that follows. We first divide the hundreds of mirror shells (see Table 1) into 20 mirror groups, such that the ratio of the largest shell radii in any two adjacent groups 
is constant. This division gives approximately equal numbers of shells in each mirror group. For each mirror group, we then use Joensen's parametrization scheme ${ }^{6}$ to specify a power-law bilayer thickness profile of the group's multilayer coatings:

$$
d(i)=\frac{a}{(i+b)^{c}}, i \in 1 . . N
$$

where the thicknesses of the topmost (thickest) and bottom-most (thinnest) layers are determined by Bragg's formula, applied respectively to the mininum and maximum energies, $E_{\min }$ and $E_{\max }$, and grazing angles, $\theta_{\min }$ and $\theta_{\max }$ :

$$
\begin{aligned}
d(1) & =\frac{a}{(1+b)^{c}}=d_{\text {max }}=\frac{h c}{2 E_{\min } \sin \theta_{\min }} \\
d(N) & =\frac{a}{(N+b)^{c}}=d_{\min }=\frac{h c}{2 E_{\max } \sin \theta_{\max }}
\end{aligned}
$$

The thickness ratio of the two materials in the multilayer is held constant throughout the profile; we indicate the thickness fraction of the high-Z material by:

$$
\Gamma=\frac{\text { thickness of high-Z material in a bilayer }}{\text { total bilayer thickness }}
$$

Note that the Bragg Equations (2) allow us to express the parameters $a$ and $b$ in the thickness profile 1 in terms of $d_{\min }, d_{\max }, N$ and $c$. Thus, $\left(d_{\max }, d_{\min }, N, c, \Gamma\right)$ constitutes a complete set of parameters to specify a multilayer profile. To determine $(N, c, \Gamma)$, we use Nelder and Mead's downhill simplex method, ${ }^{7}$ and vary these parameters to find the set that gives the highest effective area produced by the mirror group, weighted in energy and incident angle. The energy is weighted linearly:

$$
W(E)=(E+70) / 100
$$

to compensate for the fact that it is easier to obtain reflectivity at low energies at any given reflection angle. The angular weighting function weighs the off-axis angles in such a way that all pixels on the $(2.5 \mathrm{~cm})^{2}$ detector have equal weights (See Mao et $\mathrm{al}^{8}$ ). With these weighting schemes, the energy- and angle-weighted effective area becomes a workable measure of the quality of multilayer coatings.

As we have pointed out in Sect. 1, a mission for observing supernovae requires sensitivity in two energy bands that are substantially separated. Thus, for a given telescope geometry as specified in Table 1, we design an optimized set of multilayer coatings for each of the energy bands $60-85 \mathrm{keV}$ and $140-170 \mathrm{keV}$. The inner mirror groups, with their smaller incident grazing angles, are more suitable for producing high energy reflections, while the outer ones are better for the low energy band. Thus, our stategy for allocating the mirror shells to the two energy bands is as follows: Starting from the innermost mirror group, use a pure iridium coating until the maximum off-axis grazing angle of the group exceeds the critical angle for iridium at $170 \mathrm{keV}$. For each subsequent mirror group, use multilayer coatings optimized for the 140-170 keV high energy band, until the reflectivity of the multilayer coatings drops below $25 \%$ at $170 \mathrm{keV}$ at the maximum grazing angle for the group (See Fig. 1). For the remaining, outermost mirror shells, use multilayer coatings optimized for the 60-85 keV low energy band. The optimized parameters of the multilayer coatings thus produced, as well as their performance, are detailed in Sect. 3 .

\section{RESULTS}

Figure 2 shows the on-axis effective area curves of telescopes with focal lengths of 15, 20, 25 and $30 \mathrm{~m}$, corresponding to the four telescope designs in Table 1. All four designs employ W/Si multilayer coatings optimized for the energy ranges $140-170 \mathrm{keV}$ and $60-85 \mathrm{keV}$. It is immediately apparent from Fig. 2 that extending the focal length and decreasing the incident grazing angles have the positive effect of increasing the effective area, especially in the high energy band. This is mainly due to the fact that with smaller grazing angles for all shells, the reflectivity of all shells increases, and more of them can be optimized for the high energy band. Because the 

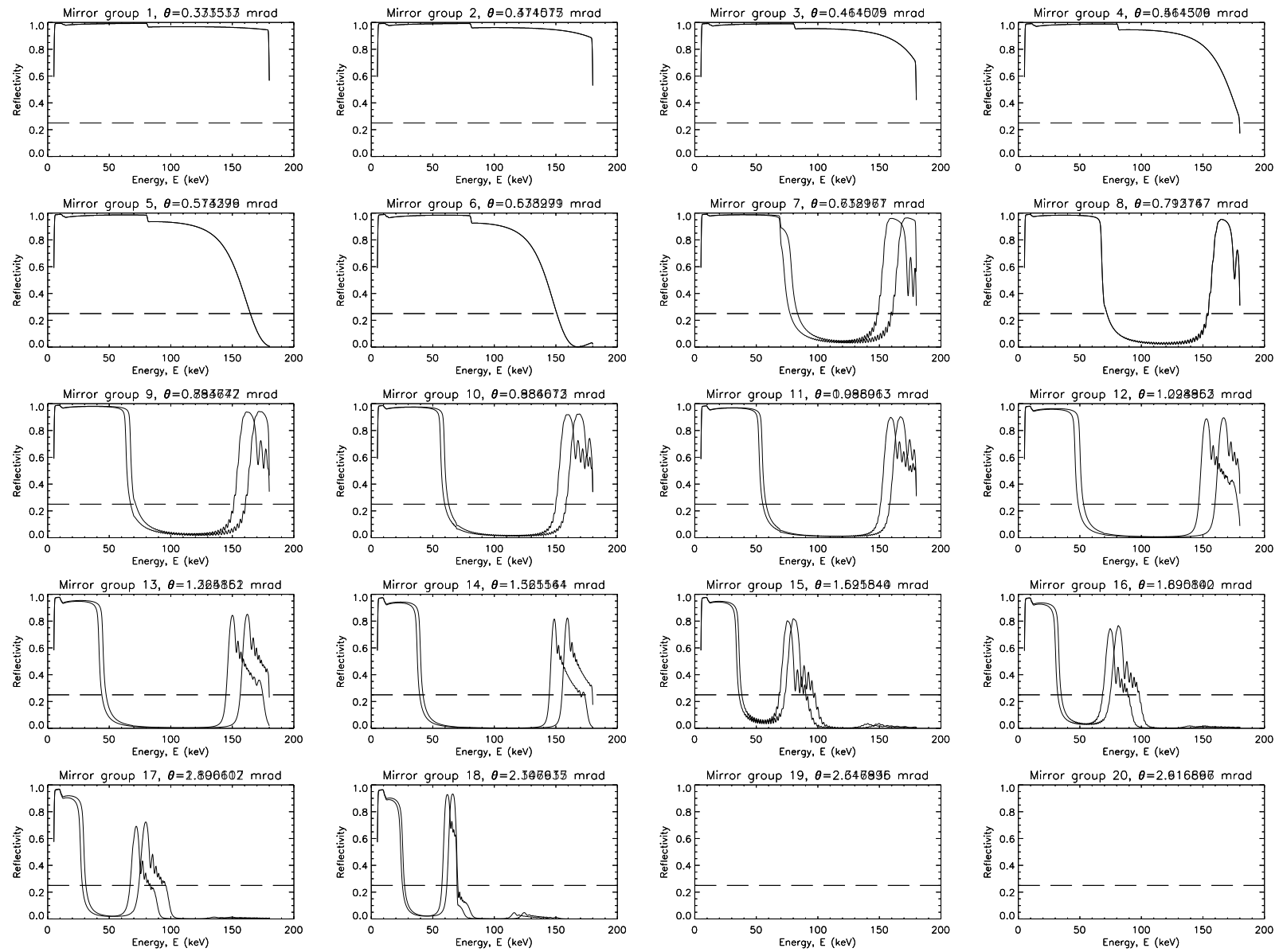

Figure 1. Typical reflectivity profile of our narrow-band multilayer coatings. Mirror groups 1-6 are coated with a single layer of iridium, groups 7-14 with graded W/Si multilayer optimized for 155-170 keV, groups 15-17 with graded W/Si multilayer for 70-85 keV, and group 18 with graded W/Si multilayer for 60-70 keV; groups 19-20 are unused. The two curves in each chart show the reflectivities at the minimum and maximum on-axis grazing angles within each mirror group; the horizontal dashed line shows the $25 \%$ reflectivity threshold which we use to determine the type of coating used in each group (see text). 


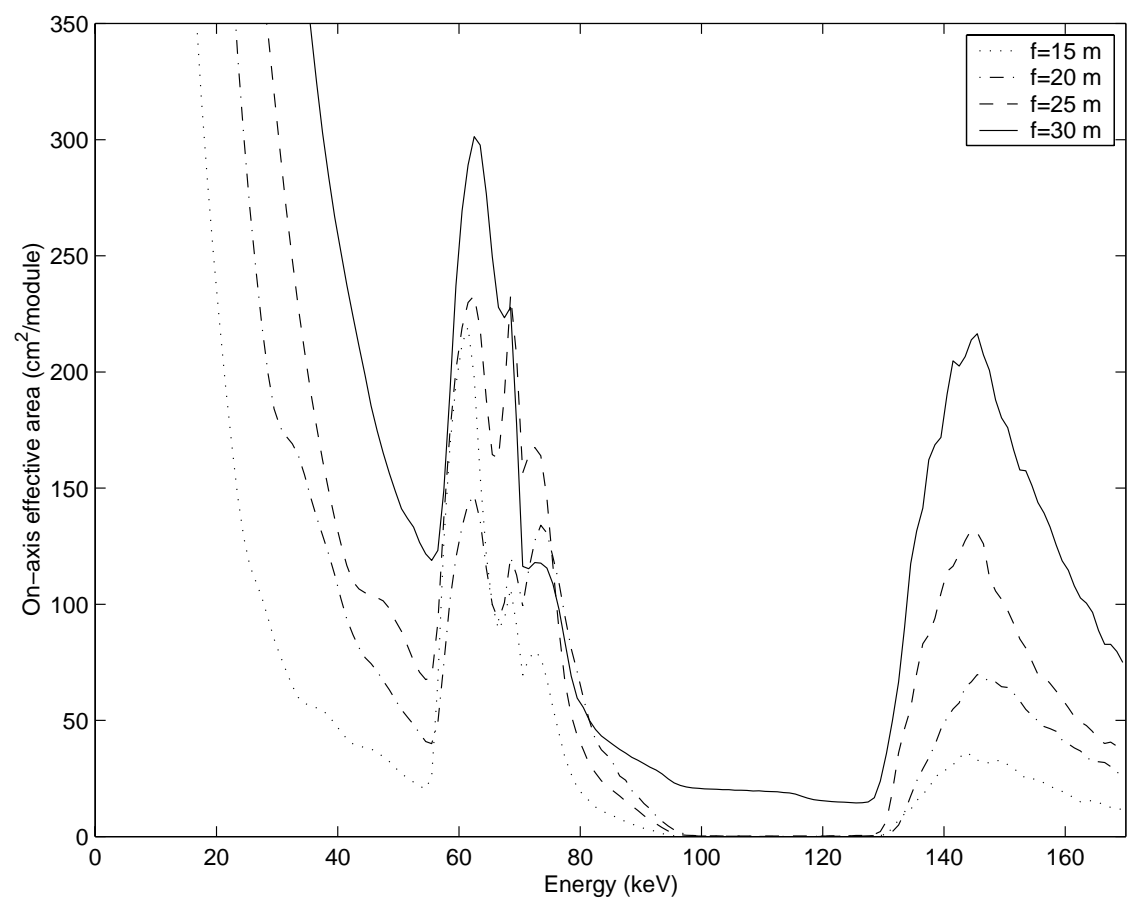

Figure 2. Dependence of the on-axis effective area on focal length. All four designs consider W/Si multilayer coatings; the geometric parameters of the telescopes are listed in Table 1. The collecting efficiency of a 5-mm CdZnTe detector is assumed in all collecting area figures throughout this paper.

multilayer coatings on each of these telescope designs are optimized for the corresponding grazing angles, they represent approximate upper limits on the maximum effective area one can obtain at the given focal lengths and radius ranges. From this comparison, we can conclude that a focal length of $30 \mathrm{~m}$ or longer is required in order to obtain a total effective area of $300 \mathrm{~cm}^{2}$ or larger over the entire high energy band with five telescope modules. A couple of other observations can be read from Fig. 2. At the low energy end of the curves, one notices that low-energy effective area below $20 \mathrm{keV}$ is obtained very much for free, because the grazing angles of most shells are below the critical angle at these low energies, and thus the reflectivity is very high. Note also that the dips common to all four designs at around $70 \mathrm{keV}$ are due to the abrupt increase in absorption in the tungsten layers around the K-edge energy (at $69.5 \mathrm{keV}$ ). In fact, in order to obtain any substantial reflectivity at all, one has to further divide the $60-85 \mathrm{keV}$ band into two, $60-70$ and $70-85 \mathrm{keV}$, and optimize separate multilayer coatings for each band.

Besides the focal length, which determines the grazing angles, the energy band of consideration also affects the reflectivity of the optimized multilayer coatings. Figure 3 shows the effect of varying the width of the high energy band on the effective area of the telescope, while keeping the focal length fixed at $25 \mathrm{~m}$ and all other geometric parameters identical. The multilayer coatings optimized for the narrower, $155-170 \mathrm{keV}$ band give a higher effective area than the broader, $140-170 \mathrm{keV}$ band within the bandwidths of interest. In a graded mulilayer coating, the thicknesses of the bilayers are spread out to provide reflectivity over a broad energy band. In this sense, having a narrower bandwidth allows the multilayer to better concentrate its reflectivity within the band. While relinquishing the capability to observe the $140-155 \mathrm{keV}$ band would provide higher effective area elsewhere, we also give up the capability to observe any ${ }^{56} \mathrm{Ni}$ emission at $158 \mathrm{keV}$ that is redshifted. Fortunately, for observations of supernovae in the local universe, this will not be a problem, as we can only see the blueshifted emission from the emitters ejected in the foreground, while those at the background are shielded from our view. 


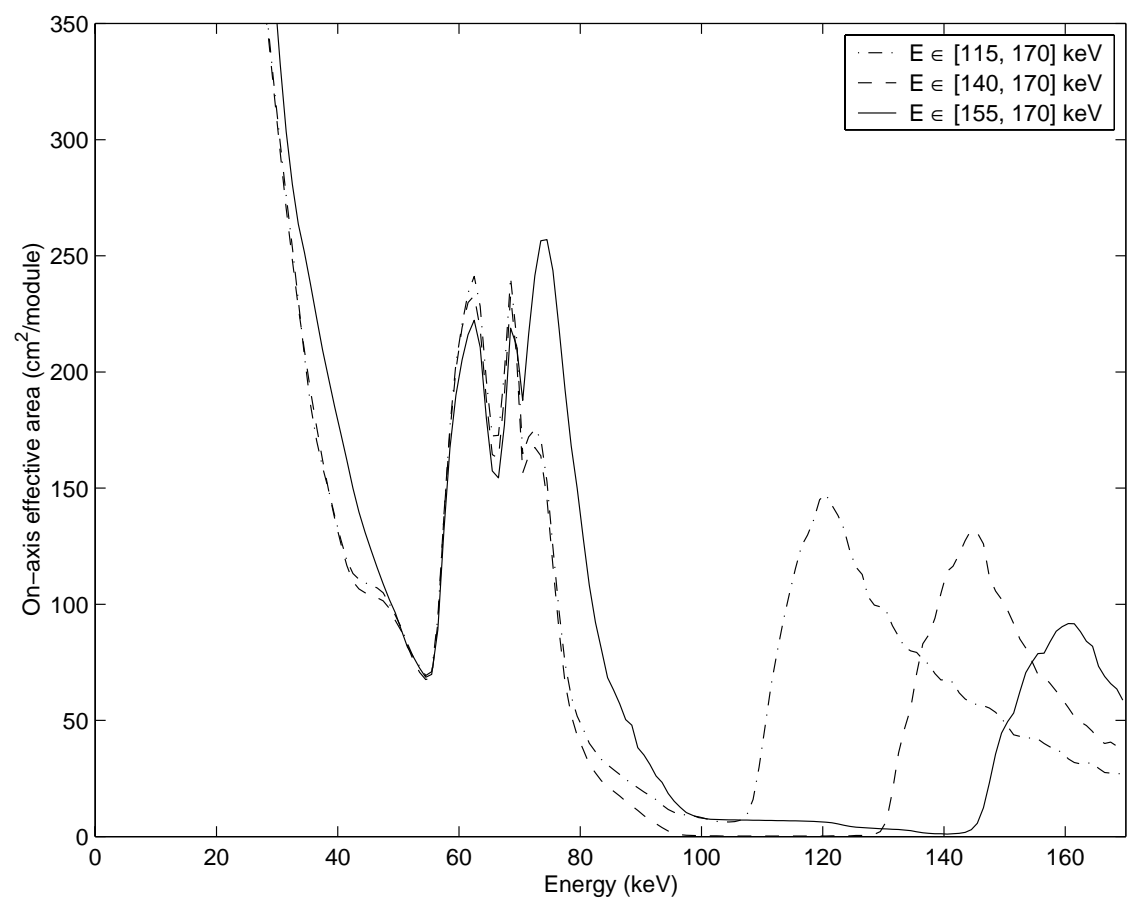

Figure 3. Dependence of the on-axis effective area on energy band. The geometric parameters of all four designs are identical, as listed in the third column of Table 1.

We have also investigated the nature of the pair of materials that make up the multilayer. Three combinations-W/Si, Pt/C and $\mathrm{Ni}_{93} \mathrm{~V}_{7} / \mathrm{Si}$ - are considered. Figure 4 shows the effective areas of telescopes employing each of these multilayer coatings by themselves, with a purely iridium-coated telescope as the reference. We have found that the $\mathrm{W} / \mathrm{Si}$ and $\mathrm{Pt} / \mathrm{C}$ combinations perform very similarly across the energy spectrum, except at the K-edges of tungsten at $69.5 \mathrm{keV}$ and of platinum at $78.5 \mathrm{keV}$. Using either pair of materials for the low energy band of 60-85 keV requires breaking the band into two at the respective K-edge energies. In contrast, $\mathrm{Ni}_{93} \mathrm{~V}_{7} / \mathrm{Si}$ coatings do not have this K-edge problem. However, there are also disadvantages in using optimized $\mathrm{Ni}_{93} \mathrm{~V}_{7} / \mathrm{Si}$ coatings. Because both nickel and vanadium have low atomic numbers, $\mathrm{X}$-ray absorption is less significant than in tungsten or in platinum; thus, X-rays can penetrate deeper into an $\mathrm{Ni}_{93} \mathrm{~V}_{7} / \mathrm{Si}$ multilayer. On the other hand, because the difference in electron densities in $\mathrm{Ni}_{93} \mathrm{~V}_{7}$ and in $\mathrm{Si}$ is smaller, the reflectance per $\mathrm{Ni}_{93} \mathrm{~V}_{7} / \mathrm{Si}$ interface is also weaker than that per W/Si or Pt/C interface; thus, many bilayers of $\mathrm{Ni}_{93} \mathrm{~V}_{7} / \mathrm{Si}$ are needed to achieve the same reflectivity as a few bilayers of $\mathrm{W} / \mathrm{Si}$ or $\mathrm{Pt} / \mathrm{C}$. As a result of both weaker absorption and reflection, an optimized $\mathrm{Ni}_{93} \mathrm{~V}_{7} / \mathrm{Si}$ multilayer coating contains over a thousand bilayers at times, which is many more than the hundreds of bilayers in an $\mathrm{W} / \mathrm{Si}$ or $\mathrm{Pt} / \mathrm{C}$ coating optimized for the same energies and grazing angles. This may pose practical problems in the coating process, as very long coating runs will be needed, while the lifetime of the raw materials and the stability of coating systems are finite. Also, a thousand bilayers amount to a coating several micrometres thick; this may pose adhesion problems or other potential issues as we tread into unexplored territories.

In practice, when one designs a telescope for a mission, one is not only concerned with the ideal telescope performance, but also limitations such as budget and weight limitations. Thus, cost and weight efficiencies may take precedence to performance. With this goal in mind, we present here a telescope design that is just sufficient in performance to achieve the scientific goals set out in Sect. 1, while the weight of the mirror assembly is minimized. From the information in Fig. 2, we know that a minimum focal length of $30 \mathrm{~m}$ is needed for the 
(a)

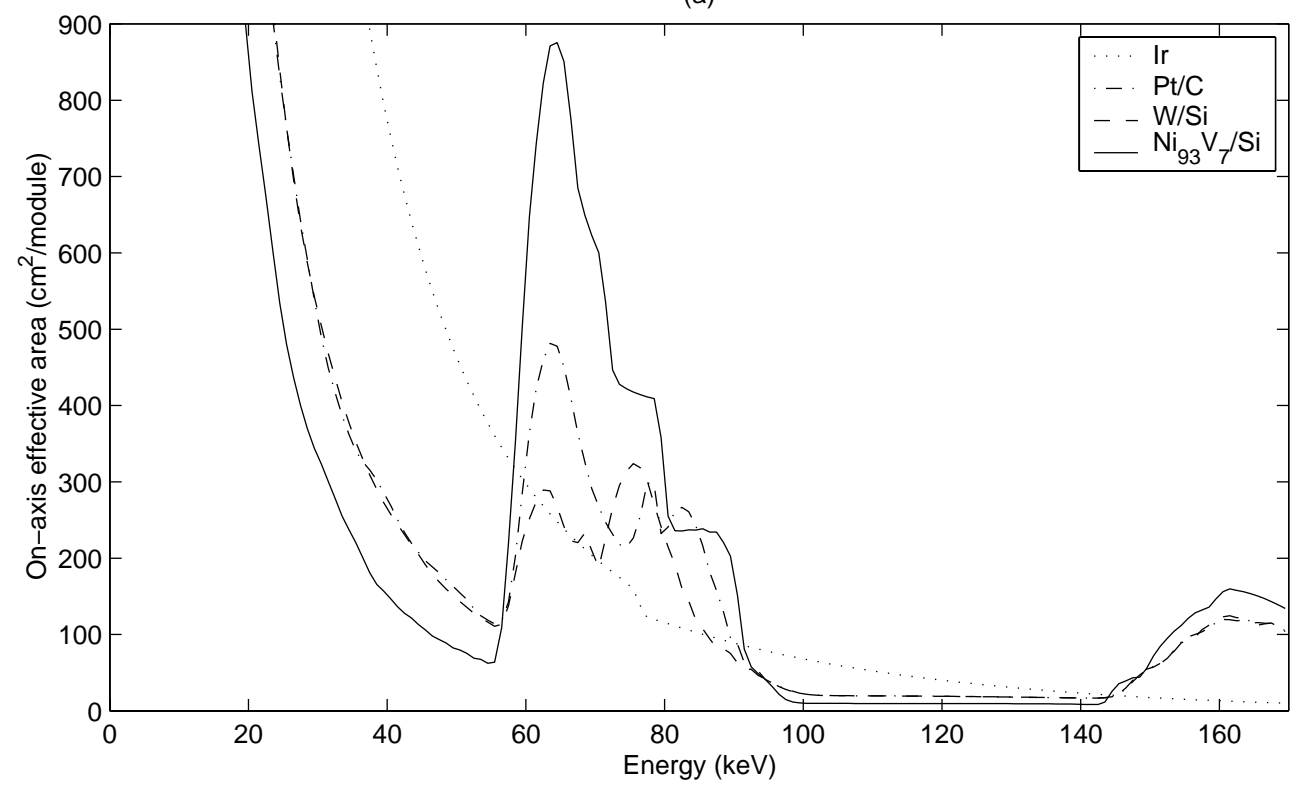

(b): Magnification of (a) for $E \in[150,170] \mathrm{keV}$

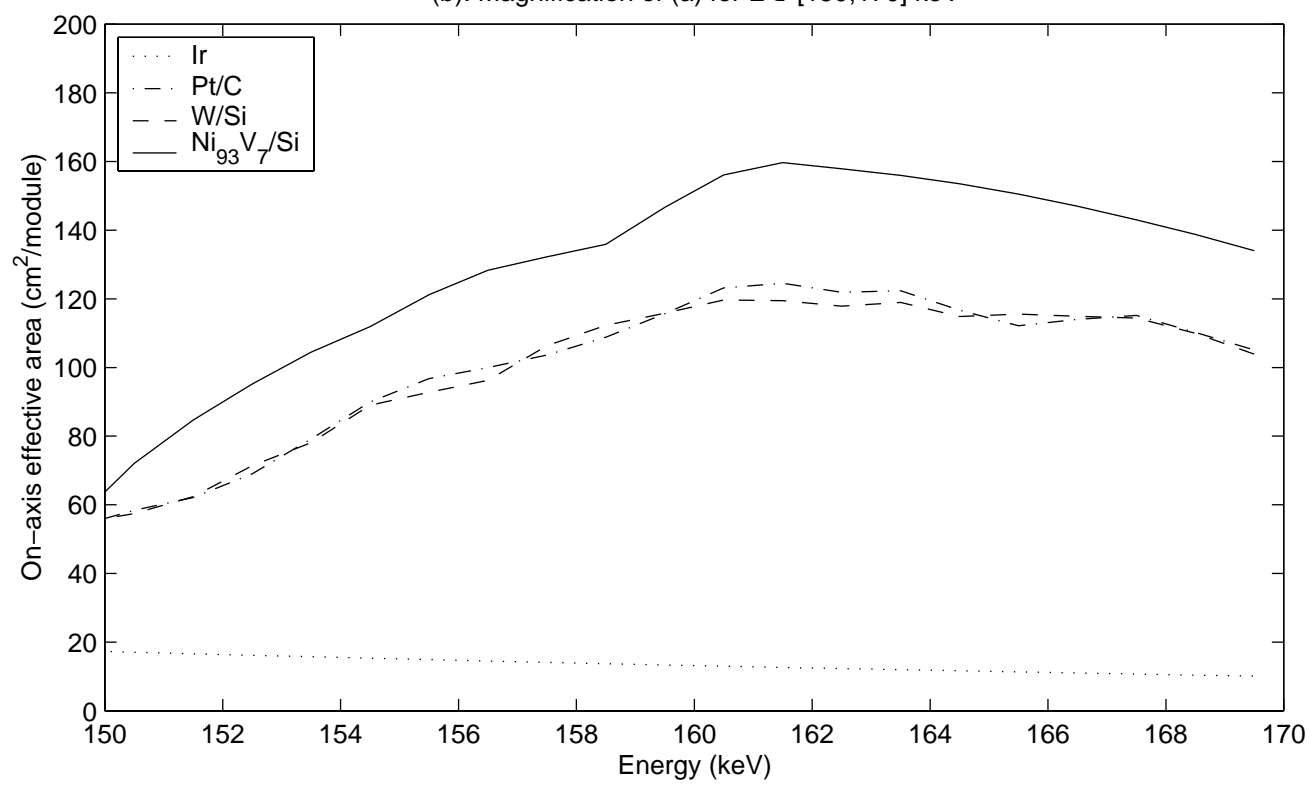

Figure 4. Dependence of the on-axis effective area on multilayer material. The geometric parameters of all four designs are identical, as listed in the last column of Table 1 . 
Table 2. Parameters for a minimum-weight telescope meeting the required scientific goals.

\begin{tabular}{|c|c|c|c|c|c|c|c|c|}
\hline Mirror group number & 11 & 12 & 13 & 14 & 15 & 16 & 17 & 18 \\
\hline Minimum shell radius $(\mathrm{cm})$ & 11.8322 & 13.1876 & 14.6982 & 16.3819 & 18.2585 & 20.3501 & 22.6812 & 25.2794 \\
\hline Maximum shell radius $(\mathrm{cm})$ & 13.1876 & 14.6982 & 16.3819 & 18.2585 & 20.3501 & 22.6812 & 25.2794 & 28.1752 \\
\hline Min. half-opening angle (mrad) & 0.986013 & 1.09896 & 1.22485 & 1.36516 & 1.52154 & 1.69584 & 1.89010 & 2.10662 \\
\hline Max. half-opening angle (mrad) & 1.09896 & 1.22485 & 1.36516 & 1.52154 & 1.69584 & 1.89010 & 2.10662 & 2.34793 \\
\hline Number of shells (total $=223)$ & 23 & 25 & 26 & 27 & 29 & 30 & 31 & 32 \\
\hline $\begin{array}{l}\text { Weight of glass }(\mathrm{kg} / \text { module }) \\
\text { (total }=86.8 \mathrm{~kg} / \text { module })\end{array}$ & 5.80 & 7.02 & 8.14 & 9.41 & 11.3 & 13.0 & 15.0 & 17.2 \\
\hline Number of modules & 10 & 10 & 10 & 5 & 5 & 5 & 5 & 5 \\
\hline Multilayer... & & & & & & & & \\
\hline Material 2 & W & W & W & $\mathrm{Ni}_{93} \mathrm{~V}_{7}$ & $\mathrm{Ni}_{93} \mathrm{~V}_{7}$ & $\mathrm{Ni}_{93} \mathrm{~V}_{7}$ & $\mathrm{Ni}_{93} \mathrm{~V}_{7}$ & $\mathrm{Ni}_{93} \mathrm{~V}_{7}$ \\
\hline Substrate & $\mathrm{Si}$ & $\mathrm{Si}$ & $\mathrm{Si}$ & $\mathrm{Si}$ & $\mathrm{Si}$ & $\mathrm{Si}$ & $\mathrm{Si}$ & $\mathrm{Si}$ \\
\hline Energy range for optimization & & & & & & & & \\
\hline minimum $(\mathrm{keV})$ & 155. & 155. & 155. & 60 . & 60 . & 60 . & 60 . & 60 . \\
\hline maximum $(\mathrm{keV})$ & 170. & 170. & 170. & 85. & 85. & 85. & 85. & 85. \\
\hline Minimum d-spacing $(\AA)$ & 33.1821 & 29.7717 & 26.7118 & 47.9329 & 43.0064 & 38.5863 & 34.6204 & 31.0622 \\
\hline Maximum d-spacing $(\AA)$ & 40.5622 & 36.3933 & 32.6529 & 75.6835 & 67.9049 & 60.9257 & 54.6639 & 49.0456 \\
\hline Interface roughness $(\AA)$ & 3.5 & 3.5 & 3.5 & 3.5 & 3.5 & 3.5 & 3.5 & 3.5 \\
\hline c & 2.487344 & 3.90409 & 3.231094 & 4.708523 & 15.644578 & 14.215713 & 15.621609 & 15.790359 \\
\hline$\Gamma$ & 0.379687 & 0.384229 & 0.400781 & 0.386584 & 0.420384 & 0.412451 & 0.443811 & 0.406311 \\
\hline Coating thickness $(\mu \mathrm{m})$ & 0.352331 & 0.494165 & 0.552642 & 0.908112 & 1.018332 & 1.421597 & 1.591227 & 2.226064 \\
\hline Figure of merit $\left(\mathrm{cm}^{2}\right)$ & 0.753039 & 0.920155 & 1.017054 & 4.291268 & 5.540548 & 6.787850 & 7.948927 & 9.045718 \\
\hline
\end{tabular}

observation of the ${ }^{56} \mathrm{Ni}$ line at $158 \mathrm{keV}$. To maximize the effective area, we choose the reduced high energy band, $155-170 \mathrm{keV}$ for our multilayer optimization, together with the $60-85 \mathrm{keV}$ low energy band. For each energy band, a set of optimizations are calculated for each of the multilayer material pairs mentioned in Sect. 3 above, and the pair producing the highest reflectivity is chosen. The resulting telescope design gives the highest effective area within the weighted angular range for a $30 \mathrm{~m}$ focal length, with fairly optimal energy bands, multilayer materials and bilayer thickness profiles. Then, we 'peel' off high energy shells from the inside of the mirror assembly, group by group until the minimally acceptable effective area within the high energy band is attained. We also peel off the low energy shells from the outside similarly to obtain the minimally acceptable effective area within the low energy band. Finally, we weigh the options of having seven equal-size modules versus having five large and five small ones (with different number of shells peeled off from the outside of each module), as stated in Sect. 1 above. For this specific design, the area requirement in the high energy band is substantially higher than that in the low energy band. Because only the inner shells contribute to high energy reflectivity, the latter option allows us to increase the ratio of high- to low-energy shells, thus meeting the area requirements with even less weight. This way, a minimum-weight telescope that meets the scientific requirements is produced. Its geometric and multilayer parameters are listed in Table 2, and the effective area is shown in Fig. 5.

Through the design process of this soft-gamma-ray telescope, we have made a couple of observations that, though not directly related to the telescope designs, are still worth mentioning. First, we note from Table 2 that the power law indices, $c$, of the bilayer thickness profiles are very much larger than the values we have encountered in the past when optimizing multilayer coatings for wider energy bands. According to Eq. (1), a large power-law index gives a thickness profile that is close to linear. This indicates that linear gradation is perhaps more appropriate for narrow band mirrors. Secondly, we note that as we focus beyond $100 \mathrm{keV}$, compton scattering becomes a significant effect. We take this into account when producing the results in this paper by making corrections to the optical constants of the multilayer materials above their respective K-edge energies. Windt et $\mathrm{al}^{9}$ in these same conference proceedings has further details of the corrections.

\section{CONCLUSION}

We have demonstrated in this paper that current technologies in multilayer X-ray focusing telescopes have now made it possible to produce a telescope that enables breakthroughs in supernova research with a mid-size 

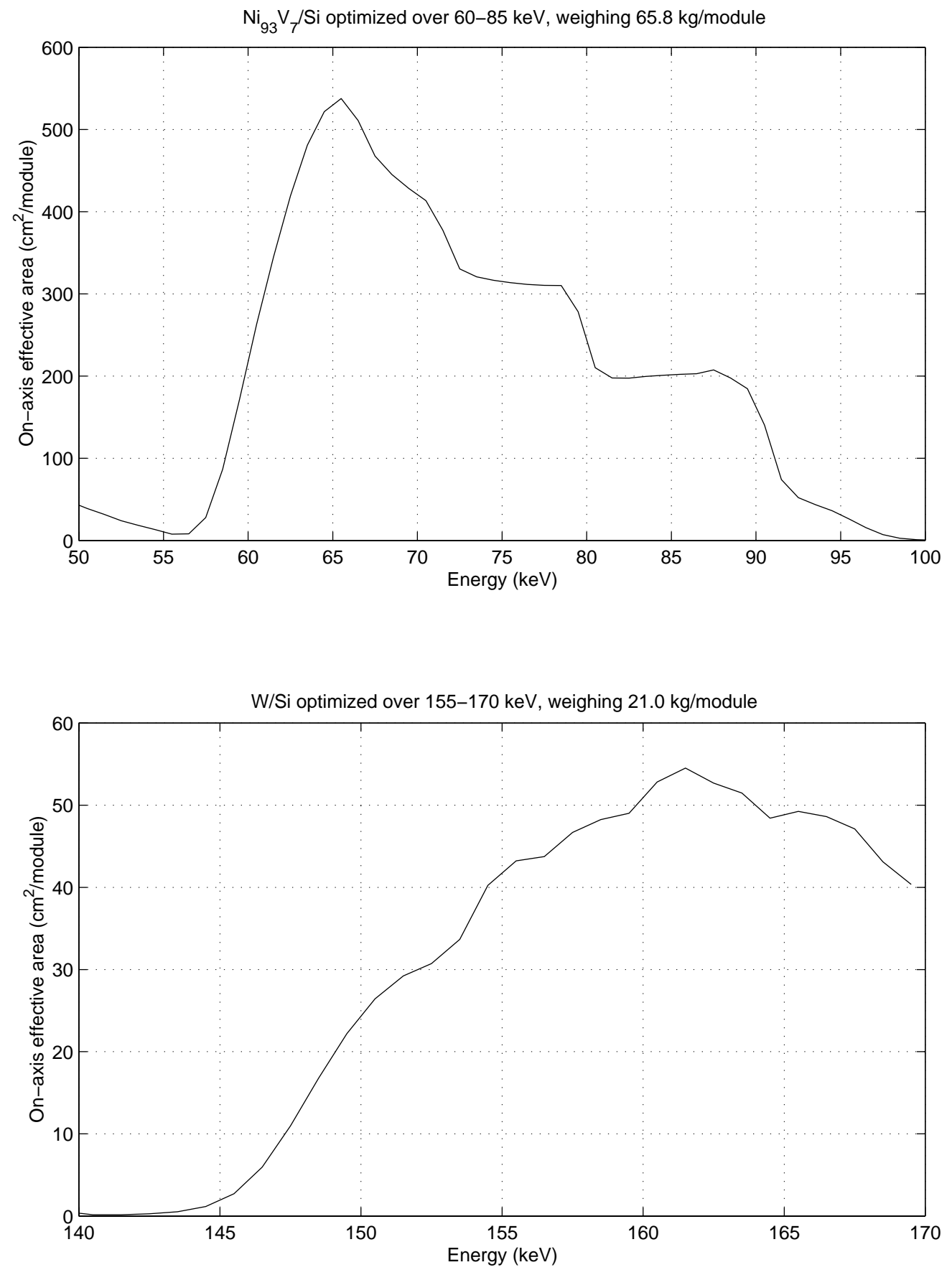

Figure 5. The on-axis effective area of the minimum-weight telescope design. See Table 2 for the geometric parameters of the telescope. 
explorer scale satellite mission. We have also exemplified how one can make the design of multilayer telescopes more versatile by mixing and matching multilayer materials, energy bands, and similar design parameters.

\section{ACKNOWLEDGMENTS}

The research reported in this paper is supported by NASA's Supporting Research and Technology (SR\&T) grant NAG5-5289. The multilayer optimization programme was run in part using the Hewlett-Packard V2500 computer system operated by Caltech's Center for Advanced Computing Research (CACR). Access to this system was provided by Prof. Thomas A. Prince. We are very grateful for all their support.

\section{REFERENCES}

1. F. A. Harrison, S. E. Boggs, A. Bolotnikov, F. E. Christensen, W. R. Cook, W. W. Craig, C. J. Hailey, M. Jimenez-Garate, P. H. Mao, S. E. Schindler, and D. L. Windt, "Development of the High-Energy Focusing Telescope (HEFT) balloon experiment," in X-Ray Optics, Instruments, and Missions III, J. E. Trümper, and B. Aschenbach, ed., in Proc. SPIE 4012, pp. 693-699, 2000.

2. K. Yamashita, P. J. Serlemitsos, J. Tueller, S. D. Barthelmy, L. M. Bartlett, K. W. Chan, A. Furuzawa, N. Gehrels, K. Haga, H. Kunieda, P. Kurczynski, G. Lodha, N. Nakajo, N. Nakamura, Y. Namba, Y. Ogasaka, T. Okajima, D. Palmer, A. Parsons, Y. Soong, S. M. Stahl, H. Takata, K. Tamura, Y. Tawara, and B. J. Teegarden, "Supermirror hard X-ray telescope," Appl. Opt. 37, pp. 8067-8073, 1998.

3. F. A. Harrison, S. E. Boggs, H. C. Chen, F. E. Christensen, W. W. Craig, N. A. Gehrels, J. E. Grindlay, C. J. Hailey, P. Pinto, S. Thorsett, J. Tueller, and S. E. Woosley, "High-resolution spectroscopic imaging mission (HSI)," in X-Ray and Gamma-Ray Telescopes and Instruments for Astronomy, J. E. Trümper, and H. D. Tananbaum, ed., Proc. SPIE 4851, 2002.

4. F. E. Christensen, J. M. Chakan, F. A. Harrison, S. E. Boggs, P. H. Mao, T. Prince, W. W. Craig, C. J. Hailey, and D. L. Windt, "Grazing incidence optics designs for future gamma ray missions," in $X$ Ray Optics, Instruments, and Missions III, J. E. Trümper, and B. Aschenbach, ed., Proc. SPIE 4012, pp. 278-283, 2000.

5. P. H. Mao, F. A. Harrison, D. L. Windt, and F. E. Christensen, "Optimization of graded multilayer designs for astronomical X-ray telescopes," Appl. Opt. Vol. 38, No. 22, pp. 4766-4775, 1999.

6. K. D. Joensen, P. Voutov, A. Szentgyorgyi, J. Roll, P. Gorenstein, P. Hoghoj, and F. E. Christensen, "Design of grazing-incidence multilayer supermirrors for hard-X-ray reflectors," Appl. Opt. 34, pp. 7935$7944,1995$.

7. W. H. Press, B. P. Flannery, S. A. Teukolsky, and W. T. Vetterling, Numerical Recipes in C: The Art of Scientific Computing, 2nd ed., $\S 10.4$, pp. 408-412, Cambridge University Press, Cambridge, 1992.

8. P. H. Mao, L. M. Bellan, F. A. Harrison, D. L. Windt, and F. E. Christensen, "Evaluation and optimization of multilayer designs for astronomical X-ray telescopes using a field-of-view- and energy-dependent figure of merit," in X-Ray Optics, Instruments, and Missions IV, R. B. Hoover, and A. B. Walker, ed., Proc. SPIE 4138, pp. 126-133, 2000.

9. D. L. Windt, S. Donguy, C. J. Hailey, J. Koglin, V. Honkimaki, E. Ziegler, F. E. Christensen, F. A. Harrison, and $\mathrm{H}$. Chen, "W/SiC X-ray multilayers optimized for use above $100 \mathrm{keV}$," in X-Ray and Gamma-Ray Telescopes and Instruments for Astronomy, J. E. Trümper, and H. D. Tananbaum, ed., Proc. SPIE 4851, 2002. 